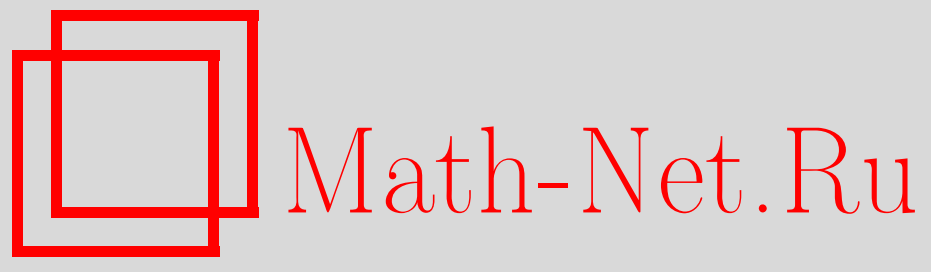

А. И. Ефимов, Заметка о зеркальной симметрии для кривых, УМH, 2010, том 65, выпуск 5, 191-192

DOI: https://doi.org/10.4213/rm9389

Использование Общероссийского математического портала Math-Net.Ru подразумевает, что вы прочитали и согласны с пользовательским соглашением http://www . mathnet.ru/rus/agreement

Параметры загрузки:

IP: 107.22 .136 .117

26 апреля 2023 г., 12:51:47

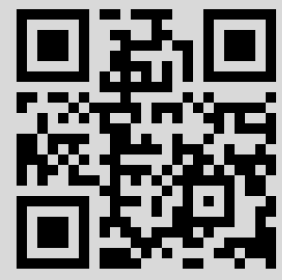




\section{Заметка о зеркальной симметрии для кривых}

\section{А. И. Ефимов}

Гомологическая зеркальная симметрия - это гипотетическая категорная интерпретация зеркальной симметрии, предложенная М. Концевичем [1]. Подход состоит в том, чтобы связать с симплектической и алгебраической стороной некоторые триангулированные категории (с $A_{\infty}$-оснащением) и доказать эквивалентность этих категорий.

Изначально гипотеза Концевича была сформулирована для зеркально симметричных многообразий Калаби-Яу. А именно, если $X$ - проективное алгебраическое многообразие Калаби-Яу, а $\widehat{X}$ - зеркально симметричное симплектическое многообразие, то с $X$ можно связать производную категорию когерентных пучков $D^{\mathrm{b}}(X)$, а с $\widehat{X}-$ $A_{\infty}$-категорию Фукаи $\mathscr{F}(\widehat{X})$. В этом случае гипотеза утверждает эквивалентность $D^{\mathrm{b}}(X) \cong D^{\pi}(\mathscr{F}(\widehat{X}))$, где $D^{\pi}(\mathscr{F}(\widehat{X}))$ - категория совершенных комплексов над $A_{\infty}$-категорией $\mathscr{F}(\widehat{X})$.

ЗАмечАниЕ 1 . Определить строго $A_{\infty}$-категорию $\mathscr{F}(\widehat{X})$ довольно непросто. В ее исходном варианте она является $A_{\infty}$-предкатегорией в смысле Концевича-Сойбельмана. Согласно доказанной автором гипотезе Концевича-Сойбельмана [2], классы эквивалентности $A_{\infty}$-предкатегорий находятся в биекции с классами квазиэквивалентности $A_{\infty}$-категорий. Это позволяет заменить $A_{\infty}$-предкатегорию Фукаи однозначно определенной $A_{\infty}$-категорией.

В данной заметке мы имеем дело с гомологической зеркальной симметрией для кривых рода $g \geqslant 2$. Более общо, гомологической зеркальной симметрии для многообразий общего типа посвящена работа [3].

Будем воспринимать такую кривую симплектически, т. е. как компактную ориентируемую поверхность $M$ рода $g \geqslant 2$ с симплектической формой. Зеркалом к ней является трехмерная модель Ландау-Гинзбурга (LG), т. е. пара $(X, W)$, где $X$ - гладкое алгебраическое многообразие, $\operatorname{dim} X=3$, и $W: X \rightarrow \mathbb{C}-$ регулярная функция.

C LG-моделью $(X, W)$ мы связываем триангулированную категорию особенностей $D_{\mathrm{sg}}(H):=D^{\mathrm{b}}(H) / \operatorname{Perf}(H)$ единственного особого слоя $H:=X_{0}$ функции $W$.

Теорема 1. Существует точная эквивалентность $D^{\pi}(\mathscr{F}(M)) \cong \overline{D_{\mathrm{sg}}}(H)$, mpиангулированных категорий, где $\overline{D_{\mathrm{sg}}}(H)$ - карубиева оболочка категории $D_{\mathrm{sg}}(H)$.

В частном случае $g=2$ эта теорема была доказана П. Зайделем [4]. Используя идеи его доказательства, удается доказать теорему для всех $g \geqslant 2$.

Опишем LG-модель $(X, W)$. Возьмем $V=\mathbb{C}^{3}$ и действие группы $K=\mathbb{Z} /(2 g+1)$ на $V$, при котором образующий элемент действует как диагональная матрица

$$
\operatorname{diag}\left(\zeta, \zeta, \zeta^{2 g-1}\right) \in \operatorname{SL}(3, \mathbb{C}), \quad \text { где } \zeta=\exp \left(\frac{2 \pi i}{2 g+1}\right) .
$$

Обозначим через $\bar{X}:=V / K$ особый фактор. У него есть каноническое крепантное разрешение $X=\operatorname{Hilb}_{K}(V) \rightarrow \bar{X}$ с помощью $K$-эквивариантной схемы Гильберта. Далее, возьмем многочлен

$$
W=-z_{1} z_{2} z_{3}+z_{1}^{2 g+1}+z_{2}^{2 g+1}+z_{3}^{2 g+1} .
$$

Он инвариантен относительно группы $K$, поэтому задает регулярную функцию на $\bar{X}$, а значит, и на $X$. Обозначим эту функцию той же буквой. Тогда $(X, W)$ - это и есть модель Ландау-Гинзбурга, зеркально симметричная кривой $M$ рода $g$.

Работа была частично поддержана Фондом поддержки молодых ученых "Конкурс Мёбиуса" и РФФИ (грант № 4713.2010.1). 
Легко видеть, что все слои $X_{s}=W^{-1}(s)$ при $s \neq 0$ являются неособыми поверхностями. Поверхность $H=X_{0}$ является объединением $(g+1)$ гладких неприводимых компонент, имеющих простые нормальные пересечения.

Схема доказательства теоремы 1 следующая. Сначала в обеих категориях выбираются генераторы $E \in D^{\pi}(\mathscr{F}(M)), F \in \overline{D_{\mathrm{sg}}}(H)$. После этого остается доказать, что $A_{\infty}$-алгебры $\mathbf{R} \operatorname{Hom}(E, E)$ и $\mathbf{R} \operatorname{Hom}(F, F)$ квазиизоморфны.

Про генератор $E$ скажем только, что это прямая сумма $(2 g+1)$ лагранжевых подмногообразий. Генератор $F$ опишем более конкретно. С помощью производного соответствия Маккея можно построить эквивалентность с эквивариантной категорией особенностей $\Phi: D_{\mathrm{sg}}(H) \cong D_{\mathrm{sg}, K}(Y)$, где $Y=W^{-1}(0) \subset V$, и $D_{\mathrm{sg}, K}(Y)=$ $D_{K}^{\mathrm{b}}(Y) / \operatorname{Perf}_{K}(Y)$. Далее, категория $D_{\mathrm{sg}, K}(Y)$ порождена объектом $\widetilde{F}:=\mathscr{O}_{0} \otimes \mathbb{C}[K]$, где $\mathscr{O}_{0}-$ структурный пучок начала координат. Тогда $F \in D_{\mathrm{sg}}(H)$ - это генератор, который соответствует $\widetilde{F}$ при эквивалентности $\Phi$. Доказательство квазиизоморфности $A_{\infty}$-алгебр эндоморфизмов генераторов использует теорию Муавра-Картана для пронильпотентных DG-алгебр Ли и теорему формальности Концевича.

\section{Список литературы}

[1] M. Kontsevich, Proceedings of the International Congress of Mathematicians (Zürich, 1994), Birkhäuser, Basel, 1995, 120-139. [2] А. И. Ефимов, “Доказательство гипотезы Концевича-Сойбельмана", Матем. сб. (в печати). [3] A. Kapustin, L. Katzarkov, D. Orlov, M. Yotov, Centr. Eur. J. Math., 7:4 (2009), 571-605. [4] P. Seidel, "Homological mirror symmetry for the genus 2 curve", J. Algebraic Geom. (to appear).

\section{А. И. Ефимов (А. I. Efimov)}

Математический институт им. В. А. Стеклова РАН

E-mail: efimov@mccme.ru
Представлено Д. В. Трещёвым Принято редколлегией 01.10 .2010 